\title{
Soil Moisture Estimation Based on GNSS-R Signal
}

\author{
Pengwei $\mathrm{Yu}^{1}$,Lei Zhang ${ }^{1,2^{*}}$ \\ 1. Engineering Center of SHMEC for Space Information and GNSS, East China Normal University, \\ Shanghai 200241, China \\ 2. Shanghai Key Laboratory of Multidimensional Information Processing, East China Normal University, \\ Shanghai 200241, China
}

\begin{abstract}
The GNSS signal which is reflected by the surface of the object carries the physical characteristic information of reflecting surface. Global navigation satellite system reflection (GNSS-R) is an emerging area of GNSS applications in the field of microwave remote sensing using multipath reflected signals. Meanwhile, using GNSS-R technology based on signalto-noise ratio (SNR) of interference signal to investigate environmental changes around the station has become a new research subject. Soil moisture estimation is one of the many potential applications of the GNSS-R technique. This paper presents our investigation of the characteristics of the GNSS interference signal. Through the experiments under the tree, interference signal before and after rainfall were analyzed and compared. It is found that interference signal frequency have changed significantly after rainfalls occurred. The experimental results show that: GNSS-R can be used to estimate the soil moisture by analyzing the frequency change.
\end{abstract}

Keywords:GNSS-R, Soil moisture, Interference signal, Lomb-Scargle spectrum analysis, SNR

\section{Introduction}

GNSS system is the most widely used navigation positioning system, it can provide high precision navigation and positioning services to global customers. GPS receiver antenna not only receives the direct satellite signal, but also receives reflected signal reflected by the surroundings. The interference of two kinds of ${ }^{\mathrm{iii}}$ signal will bring error for navigation and positioning. It is called the multipath error [1].

In many applications, reflected signals are regarded as something unwanted. But scholars in the study found the GNSS signal which is reflected by the surface of the object carries the physical characteristic information of reflecting surface. The GNSS-R technology has developed rapidly. GNSS-R does not require additional signal source transmitter, it has advantages ofhigh time resolution, converges large area, all-weather, real-time. GNSS-R makes full use of existing GNSS monitoring stations, providing a new and powerful sensor for the meteorological department [2]. And the reflecting surface features are mainly characterized by the reflection wave, polarization characteristics, amplitude, phase, frequency, and other parameters. Therefore the physical characteristics of the reflecting surface can be effectively determined by receiving and processing the reflected signal [3]. Wan et al. [4] presents a method based on multipath theory for specular reflections and the use of Signalto-Noise Ratio (SNR) data to derive local sea level variations, the correlation coefficients are better than 0.97. Scholars in domestic started relatively late in this area. Dai et al. [5,6] used GPS-MR technology based on SNR information to measure the snow depth, the correlation coefficient between GPS-MR snow depth and SNOTEL snow depth is greater than 0.95 . Wu et al. [7] proposed a method using reflected signal to measure the height of water surface by a single GPS receiver. The sea surface height derived by GPS reflected signal show excellent agreement with tide gauge measurements.

Soil moisture is an important physical quantityin hydrology, agriculture, and climate research. The information of soil moisture has the significant meanings for the monitoring of global water resources protection and utilization[8-10]. At present, there are fewdomestic studies on soil moisture in GNSS-R field.In this paper we investigated the double-antenna direction finding system, based on the achievements of above scholars. The relationship between SNR and multipath and the basic principle of GNSS-R for soil moisture technology is discussed in detail. Moreover, combined with the measured data, Power spectrum analysis is performed on the received interference signals.

\section{Principle of GNSS-R technology}

\subsection{GNSS-R system}

The GNSS satellite transmits a Right-Handed Circularly Polarized (RHCP) signal, and after reflection off the surface, the signal changes polarization to Left-Handed Circular Polarization (LHCP). The GNSS-R system consists of two antennae mounted back-to-back. One of the zenith-pointing antennae is RHCP, receiving the direct GNSS signals in the main lobe of

\footnotetext{
*Corresponding author: Lzhang@ce.ecnu.edu.cn
} 
the antenna radiation pattern. The other antenna is LHCP and nadir-looking, facing the soil surface and receiving the GNSS signals that have reflected off the surface in the antenna main lobe. Figure 1 shows the key components of the GNSS-R system and the geometry configuration in the experiments.

The antennae not only receive signals through the front or the main lobe, also receive a portion of the signals through the back or through side lobes. For example, the direct GNSS signal reaches the RHCP antenna, in addition, a portion of the reflected signal reaches the back of the RHCP antenna, interfering with the direct signal, and forms the interference signal [11]. Therefore the signal received by RHCP antenna is still interference signal.

The L-band GNSS signals have certain ability to penetrate into soil and are reflected by deeper layers when they impinge upon the ground surface, and especially are sensitive to the variation of the soil moisture. Generally speaking, two types of depth are involved. One is the penetration depth, which is defined as the depth below the surface at which 1/e of the surface power density of a perpendicularly incident wave remains in a half-space of the material at a specified frequency. The other type of depth is the 'moisture sensing depth' which is defined as the effective reflection depth of approximately one-tenth of a wavelength in the medium for passive microwave remote sensing. Though the 'moisture sensing depth' is difficult to validate experimentally, it is consistent with experimental results [12]. Here, $\Delta \mathrm{h}$ is used to represent the sensing depth, as shown in Figure 1.

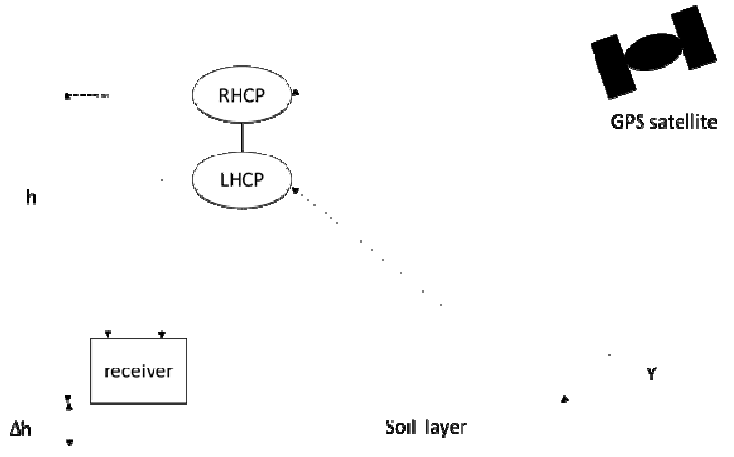

Figure 1. GNSS-R system.

\subsection{Relationship between multipath and SNR}

The signal that RHCP antenna receives is the interference signal.According to electromagnetic theory, interference signal is track by receiver PLL loop, and thesignal can be expressed as plural form $\mathrm{r}=A e^{j \varphi}, \mathrm{A}$ is signal amplitude, $\varphi$ is signal phase, $\mathrm{j}$ is the plural unit. There are [13]:

$$
\overrightarrow{r_{d}}=A_{d} e^{j \varphi_{d}}=A_{o} A_{\alpha} e^{j \varphi_{d}(1)}
$$

$$
\overrightarrow{r_{m}}=\sum_{i=1}^{m} \alpha_{i} A_{o} A_{\alpha} e^{j\left(\varphi_{d}+\varphi_{i}\right)}
$$

$$
\overrightarrow{r_{c}}=A_{c} e^{j \varphi_{c}}=A_{c} e^{j\left(\varphi_{d}+\delta \varphi\right)}(3)
$$

Where, $A_{d}$ and $\varphi_{d}$ are amplitude and phaseof direct signal respectively. And $A_{d}=A_{o} A_{\alpha} \cdot A_{\alpha}$ is called thestandard antenna gain, which is the function of satellite elevation angle, antenna gain and azimuth angle and so on. And $A_{o}$ is the fixed part of direct signal amplitude. $\alpha_{i}$ is the $i$ th satellite reflected signalamplitude coefficient relative to the $A_{d} . \varphi_{i}$ is theith satellite reflected signalphase delay relative to the $\varphi_{d} . \alpha_{i} A_{o} A_{\alpha}$ is for the reflected signal amplitude. $A_{c}$ and $\varphi_{c}$ arethe amplitude and phase of interference signal respectively. $\delta \varphi$ is the phase shiftcaused by multipath. Due to the satellite movement, $\delta \varphi$ changes over time.

The reflected signal combined with the direct signal in the receiver, forms the interference signal, which can be expressed as:

$$
\overrightarrow{r_{c}}=\overrightarrow{r_{d}}+\overrightarrow{r_{m}}(4)
$$

In the GPS receiver, tracking loop not only records the pseudorange, carrier phase, but also records carrier-to-noisedensity ratio (CNR), the ratio of signal power to the noise power spectral density, SNR is related to CNR through the noise bandwidth (B) as in SNR $=\mathrm{CNR} / \mathrm{B}$ [14], and its unit is $\mathrm{dB} \mathrm{Hz}$. It is mainly influenced by the state of receiver correlator, the antenna gain parameters, and the multipath effect. 
The SNR value is a scaled version of the signal amplitude (assuming a constant noise level). When only considered the amplitude of SNR, SNR can be used as a substitute that represent the amplitude of signal rate. SNR $A_{c}, \operatorname{SNR} A_{d}, \operatorname{SNR} A_{m}$ are the SNR of interference, direct and reflected signals, respectively. And the function can be expressed as[12]:

$$
\left.\begin{array}{r}
\operatorname{SNR}_{c}=A_{c}+\text { noise }=A_{c}=\operatorname{SNR}(5) \\
\operatorname{SNR} A_{d}=A_{d}=A_{o} A_{\alpha}(6)
\end{array}\right)
$$

The carrier tracking loop of GPS receivers presented as a phasor diagram, Figure 2 illustrating influence of multipath effects. During conditions with no multipath, thephasor diagram contains only the direct signal of amplitude $A_{d}$ and phase $\varphi_{d}$ and the SNR measurement isequivalent to $A_{d}$. With the additional signal, the receiver tracking loop attempts to track the interference signal with amplitude $A_{c}$ and phase angle $\varphi_{c}$. The interference signal is the vector sum of all phasors, containing both the direct and reflected signal, implying that the SNR is equivalent to theinterference signal amplitude $A_{c}$.

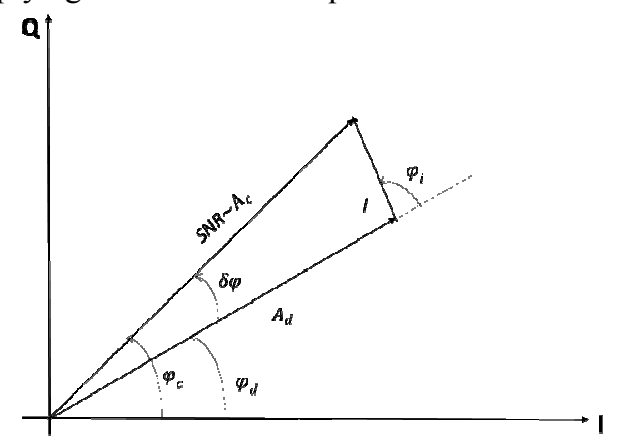

Figure 2. Influence of multipatheffects

The phase and amplitude of interference signal is affected by the phase shift and amplitude of reflected signal.With multipath present, when the phase delayof reflected signal is $\varphi_{i=} 0^{0}$, direct signal and reflected signalare in phase, the combination of them lead to the signal power increased; When the phase delay of reflected signal is $\varphi_{i=} 180^{\circ}$, direct signal and reflected signalare out phase, the power of interference signal isminimum. When the phase delay of reflected signal is $\varphi_{i=} 90^{\circ}, \delta \varphi$ get maximal value.The reflectedphase delay is vary in different times and places, so the receiving signal amplitudeand phase will appear unstable phenomenon [15].

When the multipath occurs, the satellite signal quality will be lower, the SNR value of the interference signal will show a dominant oscillation with a frequency of $2(\mathrm{~h}+\Delta \mathrm{h}) / \lambda$, when showing the SNR as a sine function of an elevation angle [4].

\section{Data processing and comparative analysis}

\subsection{The signal receiving and processing}

In order to study soil moisture, two sets of data with a period of an hour were recorded under the tree at the same time by the same receiver before and after the rainfall. This experiment we used double antenna direction finding system operating at $1 \mathrm{sample} / \mathrm{sec}$, which consists of two GPS antennae mounted back-to-back. As shown in Figure 3, two antennae were mounted on a tripod, and the zenith-pointing antennae approximately is $1.3 \mathrm{~m}$ above the soil surface.

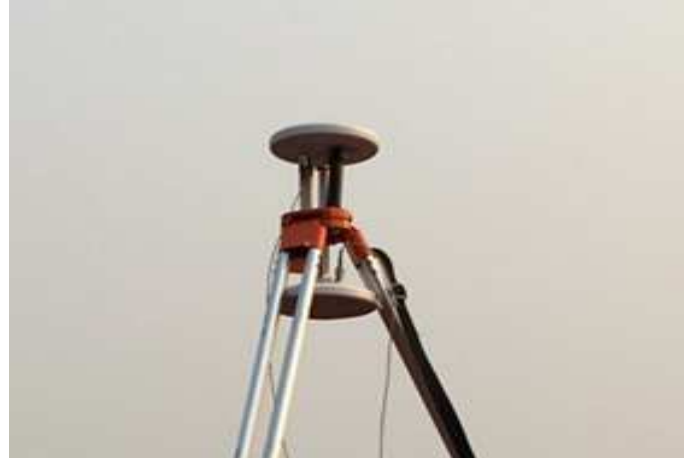

Figure 3. LHCP\&RHCP integrated antenna 
The receiver outputs the CNR and other positioning results. SNR is related to CNR through the noise bandwidth (B) as in $\mathrm{SNR}=\mathrm{CNR} / \mathrm{B}$, and its unit is $\mathrm{dB} \mathrm{Hz}$. For simplicity, CNR observation will be reported as SNR. This study only utilizes SNR observations on the L1 frequency from zenith-pointing antennae. As show in Figure 4, the L1 SNR data of PRN13, PRN20 and PRN29 before rain are shown varying from $20 \mathrm{~dB}$ to $45 \mathrm{~dB}$.

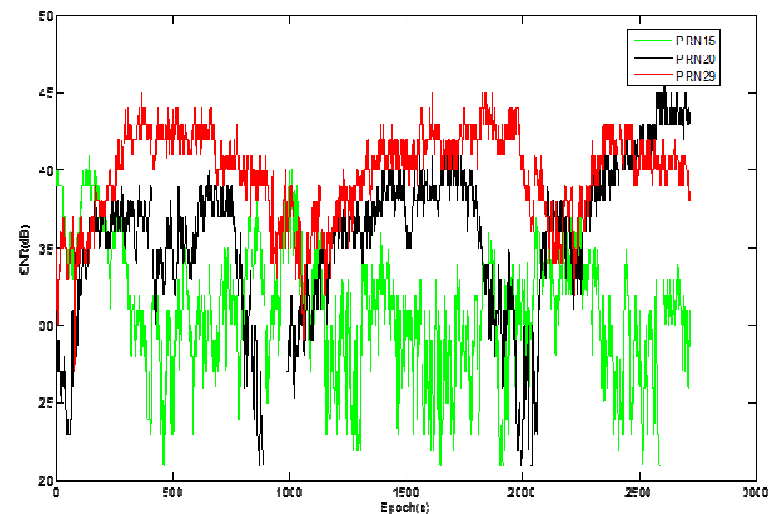

Figure 4. Raw SNR value beforerainfall.

It is necessary to separate two antenna data before processing because only the data received by RHCP antenna is needed while we used double antenna direction finding system. Then the interference signals received by RHCP antenna process to obtain information including SNR, elevation and azimuth angle. Obtain the SNR data for elevation angles between $18^{\circ}$ and $40^{\circ}$, filter the data by a smoothing filter to suppress high-frequency noise, convert decibel (dB) into amplitude (voltage) by formula

$$
\text { voltage }=10 .^{\wedge}(\mathrm{SNR} / 20)
$$

And then rearrange the amplitude as a sine function of the elevation angle. The horizontal axis in Figure 5 is the sine value of elevation angle, the vertical axis is the rearranged amplitude data after the filter is used. It can be observed that these SNR time series show a dominant oscillation when it is plotted as a sine function of an elevation angle.

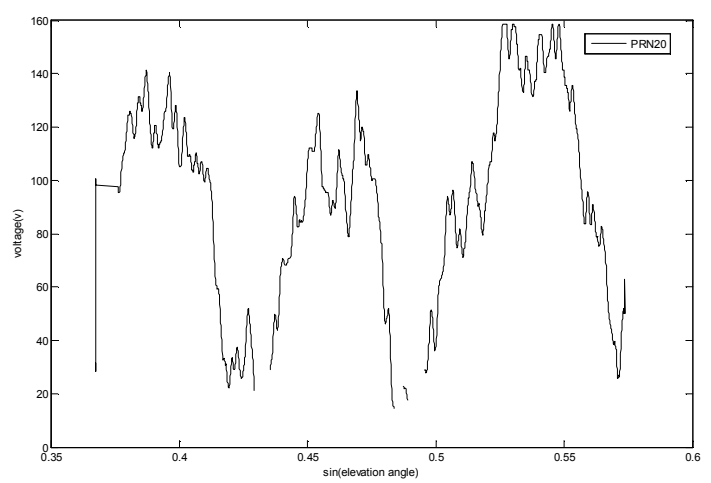

Figure 5. Amplitude in terms of voltage .

\subsection{Spectrum analysis of SNR}

The SNR data received by RHCP are evenly sampled in time, but not evenly sampled as a function of sine of the satellite elevation angle. Therefore, spectral analysis of this unevenly sampled data can be difficult for the Fast Fourier Transform. The dominant multipath frequency in the SNR data was estimated using the Lomb-Scargle spectrum analysis, also called least-squares spectral analysis, which can computes the periodogram of a sequence of unevenly spaced samples. One advantage of the Lomb-Scargle spectrum analysis over traditional Fourier techniques is that the observations do not have to be evenly sampled. 
Table 1. Difference of dominant frequency between before and after rainfall

\begin{tabular}{|c|c|c|c|}
\hline \multirow{2}{*}{ PRN } & \multicolumn{2}{|c|}{ Dominant frequency $(\mathrm{Hz})$} & \multirow{2}{*}{ Difference } \\
\cline { 2 - 3 } & Before rainfall & After rainfall & \\
\hline 15 & 9.25 & 7.91 & 1.34 \\
\hline 20 & 14.42 & 13.62 & 0.80 \\
\hline 29 & 14.01 & 13.21 & 0.80 \\
\hline
\end{tabular}

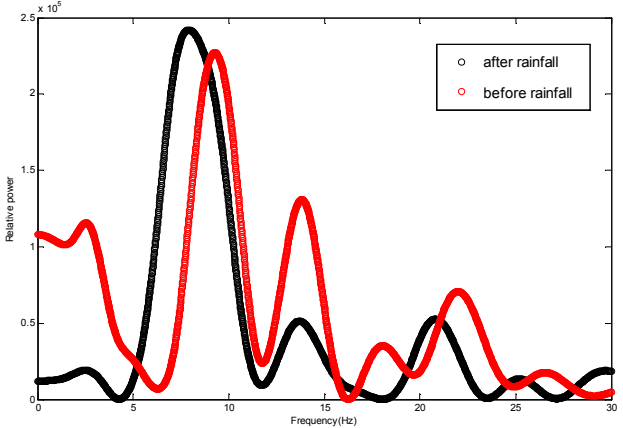

(a) PRN15

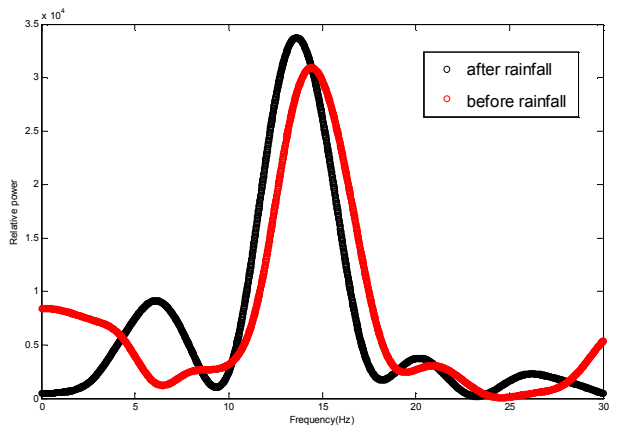

(b) PRN20

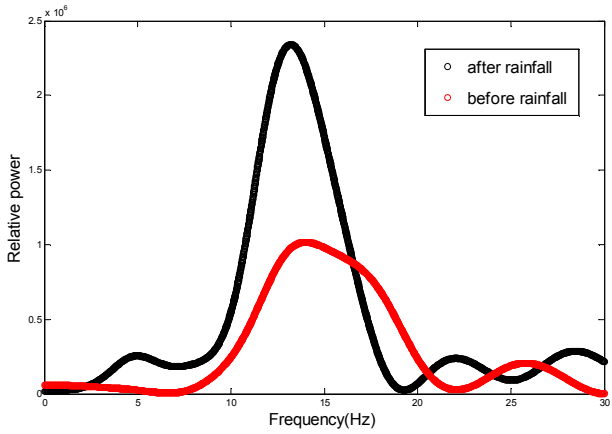

(c) PRN29

Figure 6. The Lomb-Scargle periodogramsfor SNR

Figure 6a, b, c shows Lomb-Scargle spectrum analysis of the SNR time series for the three satellites PRN15, 20, 29 received by RHCP before (red circle) and after (black circle) the rainfall. The horizontal axis shows frequency after LombScargle spectrum analysis, and the vertical axis is Lomb-Scargle spectrum amplitude of each frequency. The frequency of Lomb-Scargle spectrum in the highest peak amplitude is regarded as the dominant frequency f. Although there are clearly the dominant frequencies of the interference signals in each time series. The figures show that the powers of the interference signals are decreased and the dominant frequencies of the interference signals change after the rainfall, decrease from 9.25 to 7.91 in (a), 14.42 to $13.62 \mathrm{in}$ (b), and 14.01 to 13.21 in (c). The dominant frequencies of the interference signals decrease by about $1 \mathrm{~Hz}$ in average after the rainfall. The dominant frequencies of the interference signals are related to the antenna height (h), the effective remote sensing depth $\Delta \mathrm{h}$ (which relates to the soil moisture) and wavelength $\lambda$, which can be expressed as 


$$
\mathrm{f}=2(\mathrm{~h}+\Delta \mathrm{h}) / \lambda(9)
$$

The L-band GNSS signals are sensitive to the variation of the soil moisture. So remote sensing depth $\Delta \mathrm{h}$ decreased after rain, meaning the decrease in the dominant frequencies of the interference signals. The results indicated that spectral characteristics of the interference signal were strongly correlated with soil moisture. It is a promising approach to estimate the soil moisture by analyzing the frequency change.

The zenith-pointing antennae approximately is $1.3 \mathrm{~m}$ above the soil surface. According to the formula (9), the dominant frequency of the interference signal supposed to approximately be $13.7 \mathrm{~Hz}$, while the dominant frequency of PRN15 is far below this value. The reasons of this result may be that the environment of setting is relatively complex, which were mounted under a tree and near the building, thus many noises or signals reflected from other objects instead of soil distort the waveform of the interference signals. The result affected by all kinds of error, deviating from the true value. Therefore, in practical applications, the results of multiple satellites should be integrated to retrieve soil moisture.

\section{Conclusion}

In this paper, based on the ground experiments under the tree, the relationship between the soil moisture and SNR of interference signals is discussed in detail. And Lomb-Scargle spectrum analysis method is used to investigate the frequencies change of the interference signals. The experimental results indicate that spectral characteristics of the interference signal is closely related to the soil moisture. When the soil moisture increases, the dominant frequencies of interference signals decrease. This relationship may be used to estimate the soil moisture by analyzing the frequency change.

While the research is not yet matured, the experimental results in this paper may be influenced by many factors, there are some issues that need to be dealt with when using interference signals to estimate soil moisture. Such as the noises or signals reflected from other objects instead of soil. These noises will distort the interference signals unless they can be removed.

For future research, we will investigate methods to remove all kinds of noises, and find the ways to calculate the soil moisture.

Acknowledgment This work is supported by the National Natural Science Foundation of China(Grant No. 61671264). We thank Yao Yao and Mingming Lou to provide valuable opinions and suggestions for this article.

\section{References}

[1] Li ZH, Huang JS. GPS surveying and data processing. Wuhan university press. 2010.

[2] Zhang DK, Zhang QS. GNSS reflected signal processing: fundamentals and applications.Publishing House of Electronics Industry, 2012.

[3] Ding JC. GPS meteorology and application. Beijing : China Meteorological Press, 2009.

[4] Larson, K.M., et al. Coastal sea level measurements using a single geodetic GPS receiver. J. Adv. Space Res.(2012), http://dx.doi.org/10.1016/j.asr.2012.04.017.

[5] Dai KY, Zhang SC, Zhang Q, Nan Y, Zhang YH. Snow Depth Detection with GPS SNR Observations. Science of Surveying and Mapping, 2015, 40(12): 112-124.

[6] Dai KY, Zhang SC, Li NY, Zhang YH, Nan Y. Preliminary results of snow depth monitoring based on GPS SNR. Journal of Geodesy and Geodynamics, 2016, 36(6):525-528.

[7] Wu JZ, Yang RH. Measuring water surface height by using reflected signal of geodetic-quality GPS receiver. J Geod Geodyn 32(6):135-138.

[8] Yan SH, Gong JY, Zhang SX, et al. Ground based GNSS-R observations for soil moisture. Geophys. 2011, 54(11):2735-2744.

[9] Song DS, Zhao K, Guan Z. Remote sensing of soil moiture using global positioning system on aircraft platform. Journal of Northeast Forestry University. 2007,35(5):94-96.

[10] Xu XY, Zheng NS, Tan XL. Monitoring of Soil Moisture Fluctuation in Mining Areas Based on GPS-R. Journal of Geomatics Science and Technology. 2015,32(5): 465-468.

[11] Johan S. Lofgren. Local Sea Level Observations Using Reflected GNSS Signals, Printed in Sweden, 2014.

[12] Yan SH, Li ZY, Yu KG, Zhang KF. GPS-R L1 interference signal processing for soilmoisture estimation: an experimental study. EURASIP Journal on Advances in Signal Processing 2014, 2014:107.

[13] Wu Y, Chen X, Wu C, Mitigation of muti-path effect using SNR values. Geomat Inf Sci Wuhan Univ, 2008,33(8):842-845.

[14] Joseph, A. What is the difference between SNR and C/N0?,InsideGNSS, 2010, 5(8):20-25.

[15] Xie G. Principles of GPS and Receiver Design. Beijing : Publishing House of Electronics Industry, 2009. 